\title{
Item 7b. Patient Education and Self-Management Resources: Outpatient Setting
}

\section{KARGER}

E-Mail karger@karger.com www.karger.com/crd
(2) 2019 The Author(s)

Published by S. Karger AG, Basel

Karger

Open access

This article is licensed under the Creative Commons Attribution 4.0 International License (CC BY) (http://www.karger.com/Services/ OpenAccessLicense). Usage, derivative works and distribution are permitted provided that proper credit is given to the author and the 


\section{Heart Failure Q TOOLBOX}

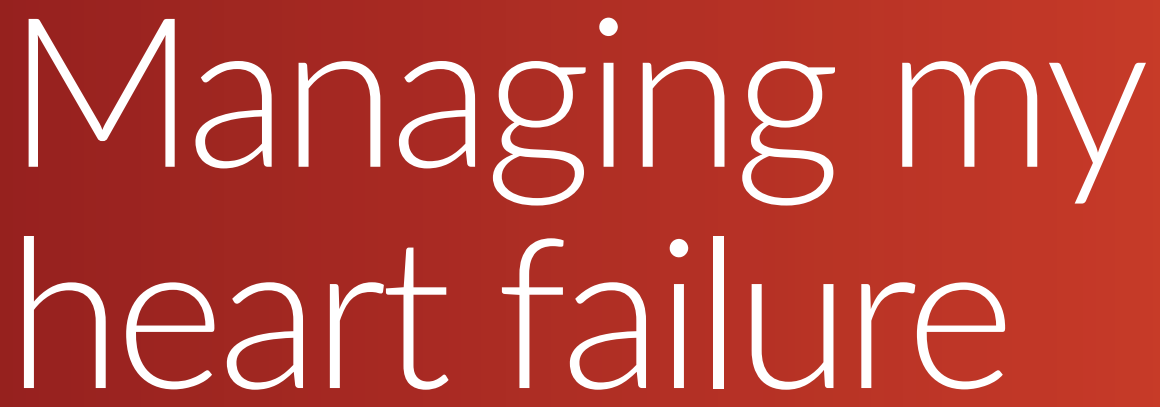

\section{Dear Patient}

The following instructions contain general information and advice regarding your condition and are designed to provide you with a guide on how best to safeguard your health. They are not intended to be exhaustive instructions.

This booklet includes the following:

- Heart failure information

- Heart failure self-management checklist

- Links to other useful resources for monitoring your heart failure 
Item 7

๑) Heart failure

The heart is a muscle that pumps blood to the body. It supplies oxygen and nutrient-rich blood to the body, which is needed for energy and growth

Heart failure occurs when the heart is unable to pump effectively to meet the body's requirements. As a result blood starts to accumulate in the lungs and fluid leaks into other areas of the body. Symptoms of heart failure include:

- Shortness of breath on exertion or at rest

- Difficulty breathing when lying flat

- Persistent coughing or wheezing

- Waking up breathless at night

- Swollen ankles, legs or abdomen

- Sudden weight gain

- Tiredness and giddiness

- Loss of appetite or nausea

- Palpitation (increased heart rate)

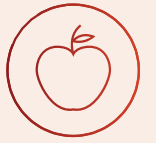

\section{Nutrition/Diet}

\section{Low sodium diet}

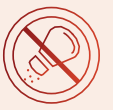

- As a patient with heart failure, it is very important to limit your salt intake, as salt causes water retention

- Many fresh foods like meat, fish, milk, vegetables and even water contain a small amount of sodium

- It is important to limit the addition of salt, soy sauce and other high salt condiments to your food

- Some easy ways to cut salt and sodium out of your food:

- Add less salt to your food. Use natural herbs and spices such as ginger, garlic, onion, lemon juice and pepper to substitute salt

- Choose fresh meat, fish and vegetables instead of processed and preserved foods

- When eating out, reduce the intake of soup/gravy

- Learn how to read food labels. Choose foods with low sodium content

- Please consult a dietitian to assist you with your meal planning 


\section{Fluid management}

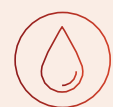

- Limit fluid intake to the amount prescribed by your doctor

- Al liquids consumed must be counted in the fluid allowance as well as food rich in fluid, for example, porridge and agar-agar

- TIP: keep a water bottle with a measurement for your own use. Use a smaller cup to control your fluid intake

\section{No alcohol intake}

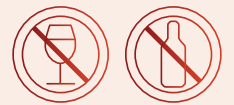

- Alcohol can cause further damage to the already weakened heart muscle

- Patients with heart failure should abstain from alcohol

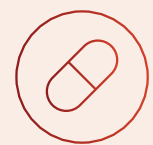

\section{Medications}

\section{Remember:}

- Do not stop taking your medicine

- It is common for your doctor to increase the dose of heart failure medicines even if you feel better because most of them work best at certain doses

\section{Safe and effective use of medications}

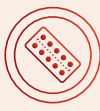

- Angiotensin-converting-enzyme inhibitor/angiotensin receptor blocker/angiotensin receptor-neprilysin inhibitor

- Reduce blood pressure

- Prolong survival and help reduce the risk of hospitalization

- Report to your doctor if you experience any persistent dry cough

- Dizziness may occur, especially during the first few days of treatment, or following an increase in dose

- If you experience any dizziness, rise slowly from a sitting or squatting position. Since several heart failure medicines may cause dizziness, your doctor may space them out at different times throughout the day

- Beta-blockers

- Reduce the heart beat and also lower blood pressure

- Prolong survival and help reduce the risk of hospitalization
- Some patients may experience transient worsening of symptoms like fluid retention or shortness of breath during the initiation of therapy, or following an increase in dose. These symptoms will usually diminish with continued use. Please monitor and report to your doctor if symptoms persist

- You might feel tired or dizzy. Rise slowly from a sitting or squatting position if you feel dizzy. Report to your doctor if unbearable

- Aldosterone receptor antagonists

- Prolong survival and help reduce the risk of hospitalization

- Report to your doctor if you experience any breast tenderness or enlargement with spironolactone (male patients)

- Diuretics

- Water pills to help remove excess fluid and salt from the body, to reduce swelling and improve breathing

- If you take furosemide twice a day, take the first dose in the morning and the second dose in the afternoon (after lunch or any time before $4 \mathrm{pm}$ ), to reduce the frequency of going to the toilet during the night

- These diuretics excrete potassium from the body. Potassium is important for maintaining a regular heart rhythm. Therefore, a blood test to determine potassium levels is undertaken periodically, to make sure that potassium levels are normal. Potassium supplement is usually given together with a diuretic

- Digoxin

- Increases the pumping ability of the heart and slows heart rate

- Please seek immediate medical attention if you notice any nausea or vomiting, blurred vision, confusion or abnormal heart rhythm

- Ivabradine

- Slows heart rate

- Improves symptoms and reduces the risk of hospitalization

- You might experience some blurred vision or have brief moments of increased brightness, most often caused by sudden changes in light intensity. These are usually mild and transient. Please report to your doctor if symptoms persist or are unbearable 
Item 7

- Vasodilators (hydralazine plus isosorbide dinitrate or isosorbide mononitrate)

- This combination of medicines prolongs life, reduces hospitalization and improves heart failure symptoms

- They relax the blood vessels and increase oxygen supply to the heart

- Flushing, headache or giddiness are usually transient and are more significant at initiation or following an increase in dose. Side effects usually diminish with continued use. Please inform your doctor if symptoms persist or are unbearable

- Please do not take sildenafil (Viagra), tadalafil (Cialis) or vardenafil (Levitra) when you are taking isosorbide dinitrate or mononitrate

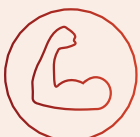

\section{Activity/rehabilitation}

- Avoid lifting and carrying heavy loads before checking with your doctor or physiotherapist

- Start with short periods of easy activity, gradually progressing to longer periods of more difficult activities

- Follow the recommended exercises, as taught by the physiotherapist during hospitalization

- Walking is the safest form of exercise and it helps to improve your stamina and symptoms. Vigorous exercises are not advisable

- If tolerable, start with walking exercises for 10 to 15 minutes, three to five times weekly

- Always monitor for symptoms of chest discomfort/pain, dizziness, breathlessness or cold sweat during walking exercises

- Consult your doctor/physiotherapist before starting other types of exercises

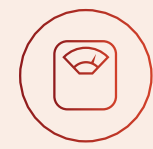

\section{Weight management}

- Get a weighing machine to check your weight

- Record your weight (in $\mathrm{kg}$ ) in a daily weight diary

\section{Heart Failure (a) TOOLBOX}

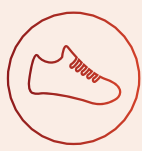

\section{Adopt a healthy lifestyle}

- Stop smoking - smoking leads to narrowing of arteries in the heart, resulting in reduced blood and oxygen supply to the heart muscle. Smoking also results in a high risk of developing blood clots and experiencing a stroke

- Keep stress under control - continuous, unrelieved stress causes your heart to work harder. Regular exercise can help to relieve stress

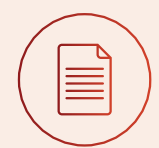

\section{When to seek further treatment}

- Seek medical consultation with your doctor if any of the following conditions occurs:

- Sudden weight gain (more than $2 \mathrm{~kg}$ within 3 days)

- Increased swelling of the abdomen, ankles, feet and legs

- Increasing shortness of breath, especially at night (when lying flat) or at rest

- Loss of appetite with bloatedness

- Constant dizziness and palpitations

- Worsening cough

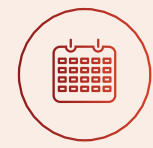

\section{Follow-up appointment}

- Regular follow-up appointments are important If you miss an appointment, remember to reschedule

- Report to your doctor on the appointment date and time

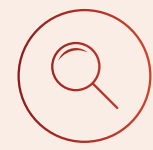

\section{Some useful educational resources on heart failure}

The following provide practical information for heart failure patients, their caregivers and family:

http://www.heartfailurematters.org http://www.heartfailure.org

HF Buddy app

(free download - please search in your app store) 


\section{Heart failure self-management}

I understand the cause of my heart failure

I have all of the medications prescribed to me and understand how to take them

I know which over-the-counter medications to avoid

I understand to weigh myself daily and to call my doctor if I experience a weight gain of more than $2 \mathrm{~kg}$ within 3 days

I can identify signs and symptoms of worsening heart failure and know what to do if symptoms worsen

I understand how to follow a low salt diet (less than 2 grams per day)

I am aware of the resources available to help me quit smoking (if I smoke)

I know the exact date and time of my next follow-up appointment 
Item 7

\section{Other useful resources for monitoring your heart failure}

\section{Warning signs information sheet}

- It is important to monitor your symptoms regularly

- The warning signs information sheet can act as a reminder of the symptoms that you should be aware of and what you should do if they occur

- The document can be found here: https://www.heartfailurematters.org/static_file/HeartFailureMatters/Documents/EN/Warning_signs.pdf

\section{Symptom and event diary}

- The symptom and event diary can be used to record any changes in your symptoms, and to document specific events relating to new or worsening symptoms. This can then be taken with you when you visit your doctor or nurse, to discuss any changes

- The symptom and event diary can be downloaded here: https://www.heartfailurematters.org/static_file/HeartFailureMatters/Documents/EN/Symptoms_and_events_diary.pdf

\section{Signs chart}

- The signs chart can be used to help you measure and record your weight, blood pressure and heart rate on a regular basis. The chart can then be taken with you when you visit your doctor or nurse, to discuss any changes. However, if you note large changes in your weight, blood pressure or heart rate, you should discuss these with your healthcare team as soon as possible

- The signs chart can be found here: https://www.heartfailurematters.org/static_file/HeartFailureMatters/Documents/EN/Monitoring\%20chart.pdf

\section{Appointment record}

- Keeping an appointment record can help remind you of upcoming appointments with your healthcare team

- A useful template, with space to note the dates, times and locations of appointments, as well as reminders of anything you need to do before your appointment, or bring with you, can be downloaded here:

https://www.heartfailurematters.org/static_file/HeartFailureMatters/Documents/EN/Appointment_record.pdf 
Heart Failure Toolbox | Outpatient Setting 


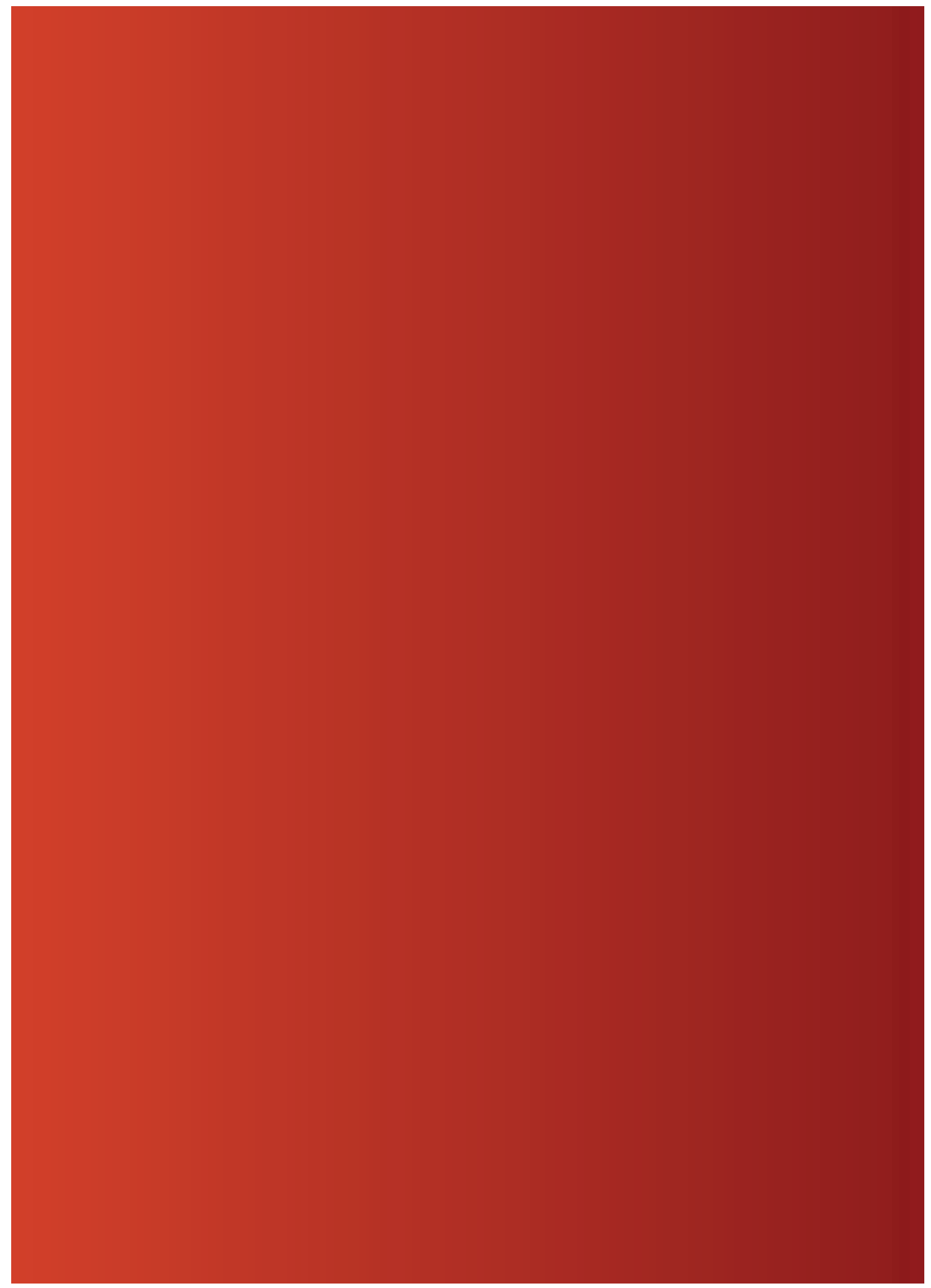

